\title{
Komplementärmedizin nach 20 Jahren Erfahrung
}

\section{Cesar Winnicki}

Der Autor dankt folgenden Personen für die Durchsicht des Textes und kritische Bemerkungen: Peter Baumann, Unternehmer, Fürigen; Dr. med. Lorenz Fischer, Dozent für Neuraltherapie, Universität Bern, KIKOM; Prof. Dr. Christian Hart Nibbrig, Literaturwissenschaftler, Epesses.
Korrespondenz:

Dr. med. Cesar Winnicki

Aeskulap-Klinik

Gersauerstrasse 8

CH-6440 Brunnen

Tel. 0418254747

Fax 0418254965

cesar.winnicki@aeskulap.com
«Jede neue Idee durchläuft drei Entwicklungsstufen: In der ersten wird sie belacht, in der zweiten bekämpft, in der dritten ist sie selbstverständlich.»

(Arthur Schopenhauer)

Komplementärmedizin ist definitiv mehr als nur ihre Methoden. Im Verständnis der Komplementärmedizin stellt Gesundheit ein labiles Gleichgewicht dar, Krankheit ist ein multifaktorielles Geschehen, und in der Folge kann eine nachhaltige Heilung nur durch Vermittlung regulativer Reize erreicht werden. Die neuste Entscheidung des Gesundheitsministers Didier Burkhalter, fünf wichtige Methoden der Komplementärmedizin in die Grundversicherung aufzunehmen, wird die sinnvolle Integration beider medizinischen Systeme fördern und der Komplementärmedizin die legitime Chance geben, ihre Wirksamkeit und Wissenschaftlichkeit im Praxisalltag auf die Probe zu stellen.

Die Komplementärmedizin erhitzt und spaltet die Gemüter seit Jahrzehnten. Besonders intensiv wurde die Debatte in der Schweiz im Vorfeld der Abstimmung vom 17. Mai 2009 über die eidgenössische Volksinitiative «Ja zur Komplementärmedizin» geführt. Die Berichterstattung in den Medien zeichnete sich durch starke Ambivalenz aus. Einerseits wurden verschiedene Methoden der Komplementärmedizin ausführlich präsentiert, andererseits kritisch hinterfragt und oft unkommentiert an den Kriterien der Schulmedizin gemessen. Komplementärmedizin entbehre jeglicher Wissenschaftlichkeit, habe lediglich Plazebowirkung, die Integration der Komplementärmedizin ins Gesundheitssystem der Schweiz würde zu unkontrollierter Kostenzunahme führen - so die Gegner.

Die wissenschaftlichen Daten zur Komplementärmedizin wurden einseitig präsentiert und beispielsweise die Ergebnisse des «Programms Evaluation Komplementärmedizin» (PEK) kaum erwähnt. Wie kritisch man auch immer diesem Programm gegenübersteht, so hat doch PEK in einigen Bereichen interessante Resultate gebracht. Nicht nur widmen die Komplementärmediziner ihren Patienten mehr Zeit und erreichen deren höhere Zufriedenheit, sondern die entstehenden Kosten sind grundsätzlich tiefer als bei konventioneller Behandlung [1].

Trotz aller Kritik, Belehrung und Einwände wurde die Initiative «Ja zur Komplementärmedizin» mit einer klaren $2 / 3$-Mehrheit angenommen, was das Diskussionsklima um die Komplementärmedizin positiv beeinflusst hat. Das Feilschen um die Positionen wich der sachbezogenen und realistischen Betrachtung. Die Schweizerische Ärztezeitung hat vor kurzem in diesem Sinne einen Artikel publiziert, dessen Autor - notabene ein Facharzt für Chirurgie dazu aufruft, den Glaubenskrieg zwischen beiden Richtungen zu beenden und eine fruchtbare Komplementarität beider Systeme zum Wohle unserer Patienten anzustreben [2]. Die neuste Entscheidung des Bundesrats, wichtige Methoden der Komplementärmedizin in die Grundversicherung zu integrieren, bereitet eine solide Basis sowohl für den weiteren Dialog zwischen den Protagonisten der konventionellen und komplementären Medizin als auch für die praktische Annäherung beider Systeme. Darüber hinaus erhält die Komplementärmedizin eine berechtigte Chance, ihre Wirksamkeit, Zweckmässigkeit und Wirtschaftlichkeit (WZW Kriterien) im klinischen Alltag zu beweisen.

Bezüglich des Nachweises der Wirksamkeit von komplementären Methoden hat das Bundesgericht in seinem Urteil BGE 123 V 53 mit ausführlicher Begründung entschieden:

- die Wirksamkeit muss wissenschaftlich, nicht aber durch Doppelblindstudien der Schulmedizin, nachgewiesen werden;

- die Wirkung, nicht aber der Wirkmechanismus ist darzulegen;

- der Nachweis der Wirksamkeit muss vorrangig unter Alltagsbedingungen dargelegt werden.

Die Komplementarität, eine harmonische Integration von Schul- und Komplementärmedizin, wird routinemässig und zu grosser Zufriedenheit der Patienten in der Aeskulap-Klinik in Brunnen SZ umgesetzt. Vor 20 Jahren hat eine private Stiftung das Zentrum für Ärztliche Ganzheitsmedizin mit dem Ziel gegründet, die ärztliche Komplementärmedizin zu fördern und mit konventioneller Medizin zu kombinieren. Vor allem aufgrund der Nachfrage der Patienten entwickelte sich diese Institution zu einem mittelgrossen Unternehmen mit 140 Mitarbeitenden, davon 25 Ärzte. Sowohl ambulant als auch stationär werden Patienten vor allem mit chronischen Leiden unter sinnvoller Berücksichtigung beider medizinischer Richtungen betreut. Zu den wichtigsten Kernkompetenzen der Aeskulap-Klinik gehören Integrative Onkologie, Behandlung von chronischen Schmerzen, Psychosomatik sowie ganzheitliche Zahnmedizin (Oralmedi- 
zin). Das fundierte, duale Konzept der Klinik in Verbund mit einer individuellen Betreuung hat eine wachsende Anerkennung und Vernetzung zur Folge. Als Beleg dafür können nicht nur die regelmässige, fruchtbare Zusammenarbeit mit vielen niedergelassenen Kollegen, sondern auch mehrere bilaterale Verträge mit grossen Schweizer Versicherern angeführt werden, sowie die FMH-Anerkennung als Ausbildungsstätte für Allgemeinmedizin.

Aus dem Blickwinkel dieser pragmatischen Erfahrung mit der Komplementärmedizin lassen sich einige essentielle Fragen formulieren. Was wollen unsere Patienten tatsächlich von uns Ärzten oder unserer Medizin? Was hat die Komplementärmedizin zu bieten, das sie so attraktiv für unsere Kundschaft macht?

\section{Der vergessene Patient}

$\mathrm{Zu}$ Beginn des neuen Millenniums führte die Schweizerische Akademie der Medizinischen Wissenschaften SAMW bei Schweizer Patienten eine Umfrage bezüglich ihrer Wünsche und Vorstellungen hinsichtlich der künftigen Gesundheitsversorgung durch. Die grosse Mehrheit der Befragten (69\% und 58\%) verlangte nach mehr Menschlichkeit und explizit nach Komplementärmedizin. Dieses unerwartete Ergebnis stimmt nachdenklich. Obwohl die moderne Medizin unbestrittene Erfolge vorzuweisen hat, möchten unsere Patienten mehr empathische und individuelle Betreuung.

\section{Patienten stellen fest, dass das heutige Gesundheits- wesen der massiven Zunahme chronischer Leiden kaum} gerecht wird.

Eine der möglichen Erklärungen für diesen Wunsch bieten die neuesten epidemiologischen Daten. Die grösste Herausforderung, vor der die heutige Medizin steht, sind die chronischen Erkrankungen. Nach den Angaben des Bundesamtes für Statistik leiden über 50\% der Schweizer Bevölkerung an chronischen Leiden. Dieses Phänomen hat nicht nur mit der Alterung der Bevölkerung zu tun, sondern bezieht sich auf alle Altersgruppen inklusive Kinder. Im Jahr 2008 hat man in den Arztpraxen der Schweiz ca. 82 Millionen Diagnosen bei einer Bevölkerung von 7 Millionen gestellt, was die Brisanz dieser beunruhigenden Entwicklung illustriert. Unsere Bevölkerung realisiert allmählich, dass die früheren «Plagen der Menschheit» wie infektiöse Krankheiten, Säuglingssterblichkeit oder Unfälle zwar eher der Vergangenheit angehören, dafür aber neue, vor allem chronische und komplexe Erkrankungen in den Vordergrund rücken. Diese Verschiebung von epidemiologischen Schwerpunkten bedarf innovativer Therapieansätze mit multimodalem und langfristigem Charakter. Unsere
Patienten stellen fest, dass trotz der Fortschritte und immensen Aufwendungen das heutige Gesundheitswesen der massiven Zunahme chronischer Leiden kaum gerecht wird. Diese Fakten bilden die wichtigste Erklärung für den Wunsch unserer Bevölkerung nach neuen, nachhaltigen und vor allem holistischen Ansätzen in der medizinischen Versorgung.

\section{Das Anliegen der Komplementär- medizin ist die Salutogenese im Gegensatz zur Pathogenese der konventionellen Medizin.}

Der Patient sucht nicht nur eine Fachperson, sondern auch einen Partner und Berater. Es geht um symmetrische Kommunikation, gegenseitiges Verständnis, Unterstützung der positiven Veränderungen, aber auch um Begleitung in schwierigen Zeiten. «Der Wert und der Kern der Medizin liegen eben nicht im Heilenkönnen, sondern vor allen Dingen darin, dass sich da jemand eines anderen Menschen in seiner Not annimmt.» [3]. Die Weltgesundheitsorganisation (WHO) kreierte in diesem Zusammenhang den Begriff «Empowerment» und versteht darunter eine verbesserte Stellung des Patienten durch Information, Mitwirkung und Mitentscheidung in allen Behandlungsprozessen. Diese «patientenzentrierte» Versorgung ist eine der essentiellen Eigenschaften der Komplementärmedizin. Obwohl die Schulmedizin zumindest in der Theorie diesen Ansatz ebenfalls verfolgt, ist die alltägliche Wahrnehmung unserer Patienten in der technisierten Welt der konventionellen Medizin weit von diesem Ideal entfernt.

Ein gutes Beispiel für die integrative, patientenzentrierte Versorgung an der Aeskulap-Klinik bietet der Bereich Integrative Onkologie. Nach einer eingehenden Evaluation aller Fakten und Befunde werden mit den Patienten alle in Frage kommenden Behandlungsoptionen ausführlich diskutiert. Zur Verstärkung von klassischen onkologischen Massnahmen wird beispielsweise bei gewissen tiefliegenden Tumoren die wirksame loko-regionale Hyperthermie eingesetzt. Aus holistischer Sicht sind die Restaurierung des Immunsystems, die Minderung der teils sehr belastenden Nebenwirkungen der konventionellen Methoden, die begleitende Psychotherapie und die moderne Bewegungstherapie zentrale therapeutische Ziele. Die individuelle Entscheidung des Patienten wird respektiert und in einem interdisziplinären Team von Ärzten, Psychologen und Physiotherapeuten praktisch umgesetzt. Die Vorteile dieses Vorgehens sind offensichtlich und drücken sich durch höhere Zufriedenheit und bessere Lebensqualität der Patienten aus. Für den konsequenten Einsatz von Antioxidantien und insbesondere Selen im Rahmen der Chemotherapie 
zur Bekämpfung von Nebenwirkungen gibt es verlässliche wissenschaftliche Evidenz [4,5]. Wie kürzlich eine randomisierte Studie bei Patientinnen mit Brustkrebs belegte, verbessert eine enge, regelmässige psychologische Begleitung signifikant die Prognose und Überlebenszeit von dieser Patientengruppe [6]. Der positive Effekt der integrativen Betreuung lässt sich bestimmt nicht durch blosse Plazebo-Wirkung erklären.

\section{Komplementärmedizin - Das Ganze ist mehr als die Summe seiner Teile}

Sehr oft wird die Komplementärmedizin lediglich auf ihre Methoden reduziert. Es wird dabei vergessen, dass der Komplementärmedizin eine andere Betrachtungsweise der Gesundheit, Krankheit und Heilung zugrunde liegt. Zwischen der konventionellen und

\section{Abbildung 1}

Komplementärmedizinisches Modell der Entstehung einer chronischen Krankheit.

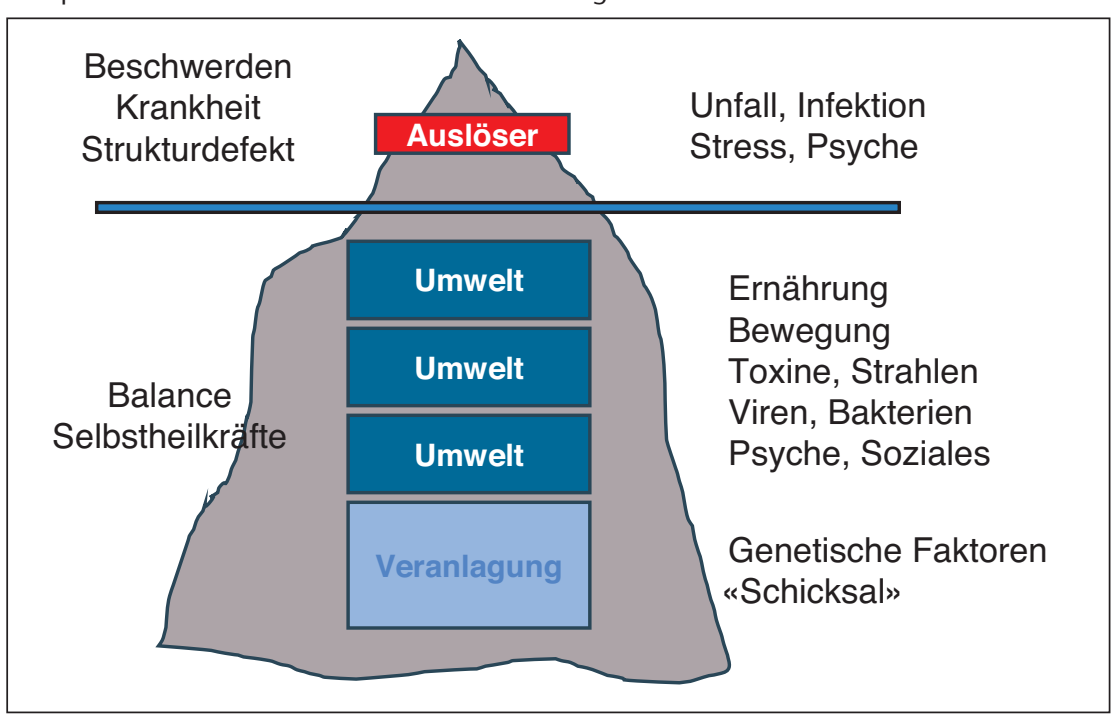

Abbildung 2

Reparativer Ansatz der Schulmedizin und regulativer Ansatz der Komplementärmedizin.

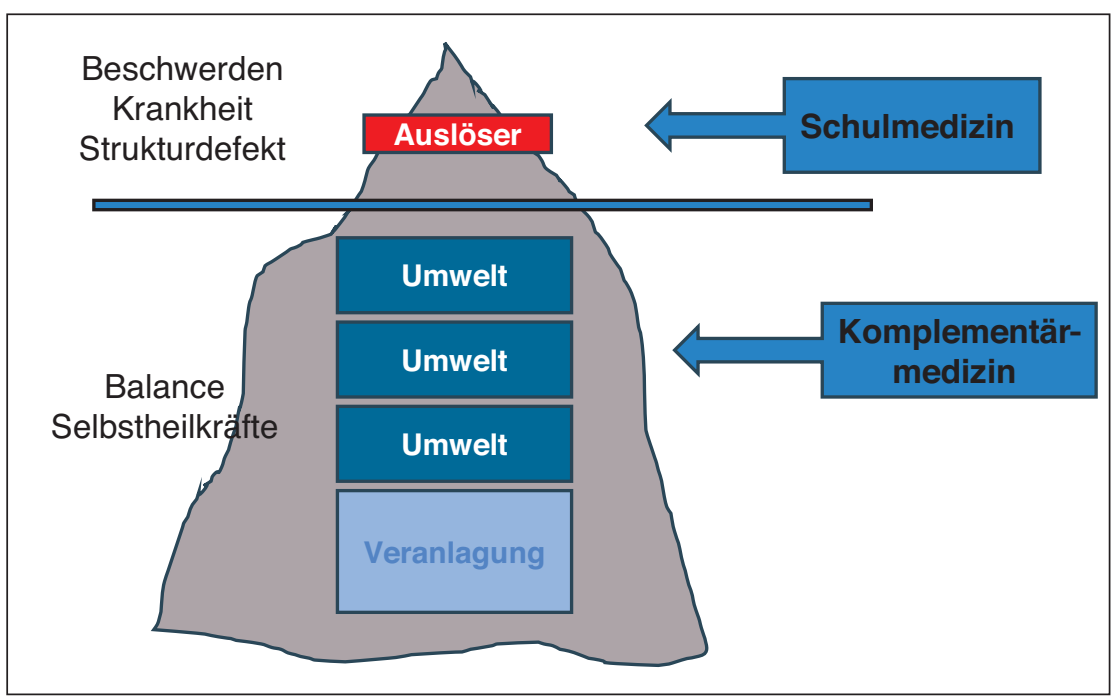

der komplementären Medizin liegen zwei grundsätzlich unterschiedliche Ansätze im medizinisch-philosophischen Verständnis der Heilung. Dies lässt sich am Modell «Entstehung einer chronischen Krankheit» gut illustrieren (Abb. 1).

Jeder chronischen Krankheit liegen genetische Faktoren zugrunde. Die zweite Schicht in der Entstehung von Krankheit stellen die vielfältigen Um-/Inweltfaktoren wie Ernährung, körperliche Aktivität, psychische Verfassung, Toxine und Infektionen dar. Ein gesunder Körper kann eine Reihe dieser negativen Einflüsse beseitigen und das labile Gleichgewicht der Gesundheit bewahren. Diese Fähigkeit, auf die äusseren Einflüsse zu reagieren, verdanken wir unseren Selbstheilungskräften nach dem Prinzip des kybernetischen Regelkreises. Solange die ganzheitliche Regulationskraft intakt ist, befindet sich unser Körper in gesundheitlichem Gleichgewicht. Leider sind die Kompensationsmechanismen nicht unerschöpflich, kleine Reize in Form einer Infektion, einer Stresssituation oder eines geringgradigen Traumas können das Gefäss zum Überlaufen bringen. Diese zum Teil wenig spektakulären Auslöser stellen die dritte Schicht in der Entstehung einer chronischen Erkrankung dar. Der zusätzliche Reiz kann nicht mehr ausbalanciert werden, der Körper meldet dies durch Beschwerden. Oft kommt es bereits in dieser Phase zu einem nicht reversiblen Strukturdefekt, wie dies z.B. beim Herzinfarkt der Fall ist.

Anhand des Modells von Abbildung 2 lässt sich etwas plakativ und vereinfacht der reparative Ansatz der Schulmedizin und der regulative Ansatz der Komplementärmedizin präsentieren.

Die Komplementärmedizin sieht ihre Rolle in der Unterstützung der Selbstheilungskräfte (Regulation) und Ausbalancierung der negativen Umwelteinflüsse. Es geht primär um die Gesundheitserhaltung, Prävention und Anregung der Regeneration. Mit anderen Worten: Das Anliegen der Komplementärmedizin ist die Salutogenese im Gegensatz zur Pathogenese der konventionellen Medizin. Ein Beispiel für das regulative Vorgehen bietet die probiotische Therapie. Die Bedeutung der Darmflora für unsere Gesundheit ist seit fast 100 Jahren bekannt. Diese Erkenntnis wurde in der komplementärmedizinischen Behandlung konsequent seit Jahrzehnten umgesetzt, und zwar nicht nur in Bezug auf gastro-intestinale Leiden, sondern auch bei diversen anderen Erkrankungen wie Allergien, Rheuma, chronischen Infektionen oder Schmerzzuständen. Die Wirksamkeit der Probiotika zumindest bei chronischen, entzündlichen Darmerkrankungen wurde in den letzten Jahren in mehreren Studien bestätigt. Wie diese Entwicklung zeigt, dürfen wir in der Diskussion um die Komplementärmedizin ihre Pionierrolle nicht vergessen. Ein weiteres Beispiel für den salutogenetischen Aspekt der ganzheitlichen Behandlung stellt die individuelle, konsequente Bewegungstherapie in der integrativen Onkologie dar. Es existiert eine Reihe von Studien, die 
aufzeigen, dass eine regelmässige körperliche Aktivität die Überlebenszeit nach einer Tumorerkrankung wesentlich verlängert [7, 8]. Die Aeskulap-Klinik erarbeitete über Jahre in der Zusammenarbeit zwischen

\section{Die Diskussion um die Komplementärmedizin wäre nie so polemisch und intensiv, wenn unsere Patienten mit der heutigen Schulmedizin zufrieden wären.}

Onkologen und Physiotherapeuten ein Konzept, wie man bei den Krebspatienten die Idee der regelmässigen körperlichen Aktivität praktisch und vor allem konsequent umsetzt.

Die Komplementärmedizin geht somit mit einem anderen philosophischen und methodologischen Verständnis sowohl des Menschens als auch seiner Krankheit einher. Durch die vernetzte, vielschichtige Betrachtung ergeben sich sinnvolle, regulative Ansätze für die individuelle Betreuung.

\section{Komplementäres Denken fördern}

Wie der Name besagt, soll in der Humanmedizin der Mensch mit all seinen Wünschen, Bedürfnissen und komplexer Identität im Zentrum des Interesses stehen. Die selbstbewussten, mündigen Patienten setzen bei ihrem Arzt nicht nur Fachlichkeit, sondern auch Menschlichkeit und Emotionalität voraus. Die Integration komplementärmedizinischer Ideen und Methoden in die konventionelle Vorgehensweise kann bestimmt helfen, diesen hohen Anspruch unserer Patienten besser zu erfüllen. Gerade weil die Systeme so grundsätzlich verschieden sind, kann eine harmonische Allianz beider medizinischen Richtungen einen wesentlichen Beitrag leisten, die künftigen
Herausforderungen des Gesundheitswesens besser und nachhaltiger zu meistern.

Die Diskussion um die Komplementärmedizin wäre nie so polemisch und intensiv, wenn unsere Kunden bzw. Patienten mit der heutigen Schulmedizin zufrieden wären. Es scheint, dass die Vorstellungen, Bedürfnisse und das Verständnis von Gesundheit, Krankheit und Heilung bei den zwei Schlüsselexponenten des Gesundheitswesens, also Ärzten und Patienten, zu weit auseinanderliegen. Die laufende, zunehmend sachliche Diskussion bietet in diesem $\mathrm{Zu}$ sammenhang die Chance einer Annäherung. Es scheint, dass die Zeit gekommen ist für eine «Generalrevision unseres medizinischen Grundverständnisses» (Frank Nager). Und dies kann nur mit gemeinsamen Kräften in einem integrativen Ansatz geschehen.

\section{Literatur}

1 Studer HP, Busato A. Ist ärztliche Komplementärmedizin wirtschaftlich? Schweiz Ärztezeitung. 2010;91(18):707-11.

2 Spinnler P. Glaubensbekenntnisse: Komplementärmedizin - Wissenschaft - Schulmedizin. Schweiz Ärztezeitung. 2010;91(11):462-5.

3 Maio G. Auf der Suche nach Sinn. PrimaryCare. 2010;10(3):43-4.

4 Whanger PD. Selenium and its relationship to cancer: an update dagger. BrJNutr. 2004;91:11-28.

5 Ladas EJ et al. Antioxidants and Cancer Therapy: A Systematic Review. JCO. 2004;Vol 22(3):517-28.

6 Andersen BL et al. Psychologic intervention improves survival for breast cancer patients. Cancer. 2008;(113):3450-8.

7 Meyerhard JE et al. Physical Activity and Survival After Colorectal Cancer Diagnosis. JCO. 2006; Vol 24(22):3527-34.

8 Holmes MD et al. Physical Activity and Survival After Breast Cancer Diagnosis. JAMA. 2005;293:2479-86. 